\title{
Differential Laser Interferometer for Nanometer Displacement Measurements
}

\author{
C.-K. Lee* \\ National Taiwan University, Taipei 10764, Taiwan, Republic of China \\ and \\ T.-W. Wu ${ }^{\dagger}$ \\ IBM Almaden Research Center, San Jose, California 95120-6099
}

\begin{abstract}
A dual beam differential laser interferometer/vibrometer measurement system was developed for studying the steady-state and dynamic behavior of low-weight high-performance mechanical systems. This newly developed optical system employs many optical and mechanical design tactics to achieve design targets such as nanometer displacement accuracy, ease of use, capability of measuring untreated structural surfaces, high-measurement bandwidths, and large-dynamic ranges. Both the optical/mechanical configurations and the design approaches adopted are discussed in detail. A flying slider and thin-film disk system currently used in the disk drive industry were used as the testbed to verify the capabilities of this newly developed nanometer structural displacement/velocity measurement system.
\end{abstract}

\section{Introduction}

TSTRUMENTS that can perform noncontact dynamic measurements for structural systems are essential for studying and evaluating structural performance. Applications of these types of instruments range from examining the performance of viscous damper or joints in nanometer displacement ${ }^{1-3}$ to measuring slider/disk spacing used in today's direct access storage devices (e.g., the hard disk drives used in personal computers). ${ }^{4-6}$ However, currently available laser interferometers ${ }^{5-7}$ require a specular reflective surface on the measurement surface as these interferometry systems will not function properly unless the wave front quality of this reflective surface is within a fraction of the laser wavelength used. This tight wave front requirement typically requires a high-quality mirror or a retroreflector to be added to the measurement surface. The weight of this added optical surface and its mounting fixture places a stringent constraint on adopting conventional interferometer systems to be used to examine general structural performances, especially the dynamic behavior of lightweight structures. As lightweight, high-performance precision structures become more and more an integral and necessary part of today's high-technology systems, whether in aerospace structures or in precision machining tools, a new and better measurement system will be needed to further advance the field of precision mechanics.

A newly developed differential laser interferometer/vibrometer that adopts the Doppler principle, phase/frequency decoding algorithm, and balanced mechanical design to achieve noncontact differential and absolute displacement/velocity measurements of opaque objects will be discussed in this paper. Unlike the traditional laser interferometer, displacement of untreated surfaces can now be measured because of the newly designed optical configuration. The fringe interpretation schemes used provide phase decoding accuracy corresponding to fractions of a nanometer displacement. A novel balanced mechanical design was also developed for flexibility in alignment and for insensitivity to interferometer arm fixture thermal deformation induced measurement noise. With this balanced mechanical design and the new optical configuration

Received Feb. 5, 1993; revision received Feb. 7, 1995; accepted for publication Feb. 21, 1995. Copyright (C) 1995 by the American Institute of Aeronautics and Astronautics, Inc. All rights reserved.

*Associate Professor, Institute of Applied Mechanics, College of Engineering. Member AIAA.

${ }^{\dagger}$ Research Staff Member, IBM Research Division, Department K64/803, 650 Harry Road. adopted, displacement resolution as high as a few nanometers have been achieved despite the noise generated from environmental factors such as air flow fluctuations, temperature effect, dynamic vibrations, etc.

One thing that should be noted is that the position signal was obtained by directly detecting the phase change induced by the relative movement of the two opaque objects and not by integrating the velocity signal typically obtained by a laser Doppler vibrometer. ${ }^{8-11}$ More specifically, since this newly developed measurement system can obtain optical signals clean enough to allow phase decoding to be performed, we can obtain displacement signals by phase decoding while obtaining velocity signals by frequency demodulation. This approach provides this newly developed system with a capability to measure true displacement and true velocity signals directly without suffering a deterioration of resolution in low-frequency (below $20 \mathrm{~Hz}$ ) small velocity regimes when a laser Doppler vibrometer velocity signal is integrated in a time domain to obtain displacement signals.

\section{System Configuration and Its Design Considerations}

The schematic layout of the newly developed differential interferometer/vibrometer is shown in Fig. 1. The primary mechanical system which will be discussed in this paper is used in today's high-performance hard disk drives and includes two measurement surfaces of which surface 1 (Fig. 1) is the thin-film disk and surface 2 is the flying slider made of aluminum oxide/titanium carbide $\left(\mathrm{Al}_{2}\right.$ $\mathrm{O}_{3} \cdot \mathrm{TiC}$ ). This type of mechanical system poses one of the most demanding measurement tasks for nanometer-type of displacement as it is lightweight and has a large common mode displacement when the disk is spinning at high speeds (typically at $60 \mathrm{~Hz}$ or higher). More specifically, the vertical runout of the disk during spinning is typically in the range of $50 \mu \mathrm{m}$ while the flying slider tries to maintain the required $100 \mathrm{~nm}$ or less relative spacing. In other words, the quantity of interest, i.e., the relative spacing, when compared to the common mode vertical runout (a noise to the measurement system) is only in the range one part in 500. The nanometer measurement accuracy requirement is approximately in the range of $1 / 500,000$. To make measurement tasks more demanding, the discrepancies on the reflectivity of the two surfaces are significant. The thin-film disk has a specular reflective surface (mirror-like), whereas the slider possesses a scattering surface. Because of the aforementioned challenging properties, any measurement system developed for this mechanical system can be easily adapted to other lightweight highperformance mechanical systems, such as those typically found in today's aerospace structures. 


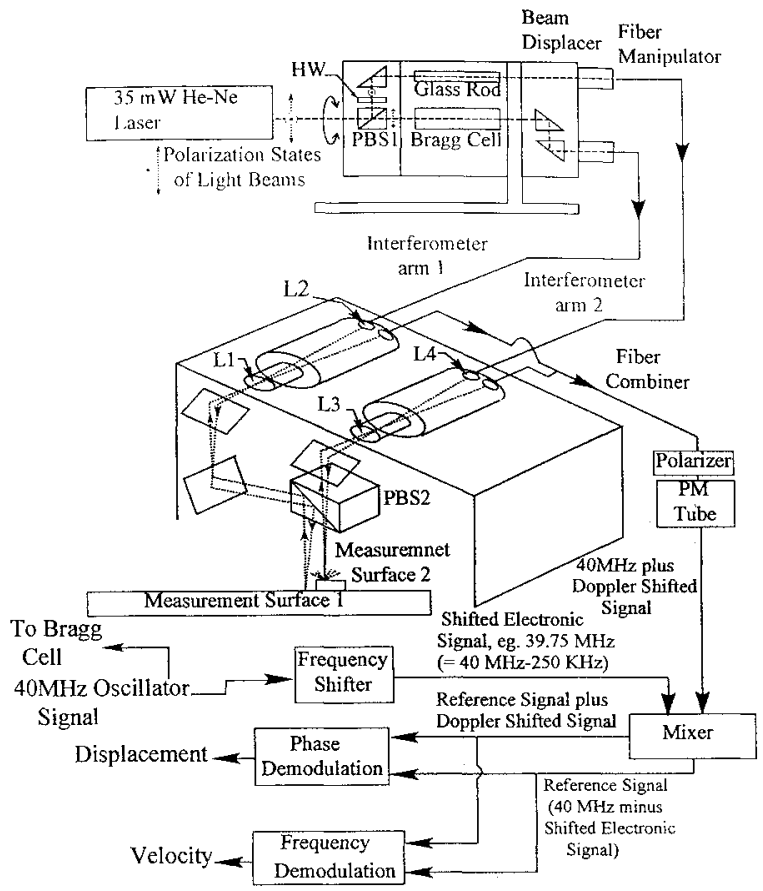

Fig. 1 Configuration of a differential interferometer/vibrometer.

To create a measurement system that requires no external calibration and has high accuracy, it was decided early on that the optical interferometry principle should be adopted since the optical wavelength can be used as the intrinsic ruler. Once an optical interferometry system was chosen as the design target, the optical configurations designed must achieve high signal-to-noise ratio for phase decoding by ensuring the two interference arms have equal intensity, the same polarization states, and uniform/good wave front quality. Since the system not only has to measure absolute displacement of a surface but also has to handle small differential displacement from two measurement surfaces that might have large common mode motions, an optical differential measurement must be done due to the electronic resolution and working range limitation imposed if the difference is to be done electronically. Taking the flying slider and disk spacing measurement as an example, the total differential spacing measurement is in the range of less than $600 \mathrm{~nm}$ whereas the runout on the disk (common mode displacement) is in the range of tens of micrometers, say, $50 \mu \mathrm{m}$. If differential measurements must be taken by measuring the absolute displacement ${ }^{11}$ separately and then performing subtraction, the measurement dynamic range to accuracy ratio is in the order of $50,000(50 \mu \mathrm{m} / 1$ $\mathrm{nm})$. However, if the differential measurement is done optically, this ratio reduces to the order of $600(600 \mathrm{~nm} / 1 \mathrm{~nm})$. The optical configuration shown in Fig. 1 achieves the design goals and many other advantageous points through the design choices discussed subsequently.

\section{Equal Intensity}

Taking the slider/disk spacing measurement as an example, the slider surface scatters with around $1 \%$ of the incident light collected by the optical lens (L2) whereas almost all incident light in the thin-film disk side (interference arm 1) is collected by the measuring optics. More specifically, in order to ensure the intensity of the two returning light beams $I_{1}^{r}$ and $I_{2}^{r}$ identical, the optical components and the laser are rotatable so that the polarization state of the laser light and the polarizing beam splitter (PBS1 in Fig. 1) can precompensate the intensity of the interference arms. That is, for the case that $I_{1}^{r}=m I_{1}^{i}$ and $I_{2}^{r}=n I_{2}^{i}$, we must have $I_{1}^{i}=(n / m) I_{2}^{i}$ to ensure $I_{1}^{r}=I_{2}^{r}$, where $m$ and $n$ are the ratios between the return light intensity and that of the incident light in interference arms 1 and 2 , respectively. With $\mathrm{n} / \mathrm{m}$ in the range between 0.01 and 100 , a commercially available polarizing beam splitter and a high-quality polarized laser can easily meet that requirement.

\section{Some Polarization States}

The half-wave plate (HW in Fig. 1), the orientation of the single-mode polarization preserving optical fibers, and the second polarizing beam splitter (PBS2 in Fig. 1) are put in place to ensure the same polarization states of the two interference arms in the photomultiplier tubes (PM in Fig. 1). Two optical fibers were fused together to form the optical fiber combiner in order to alleviate the alignment efforts required. To further reduce the polarization error noise, ${ }^{12}$ a polarizer was put in place right between the fiber combiner and the photomultiplier tube. Since the optical laser beam remained linearly polarized, a polarizing beam splitter (PBS2) can be used to place the two measurement spots anywhere from zero to several inches, i.e., the two measurement spots were separated by using the polarization state of the light beams. This flexibility in varying the separation distance is important for general structural response measurement. In the case of slider flying height measurements in hard disk drives, the disk profile and runout effect on the measured flying height (common mode noise) becomes more significant when the relative distance of the two measurement spots increases. The freedom for the user in adopting a polarizing beam splitter (PBS2) without destroying system performance also minimizes light signal loss typically found in using Mach-Zehnder interferometer type vibrometers/interferometers to measure two closely spaced objects as shown in Fig. 1. ${ }^{10,13}$ Since circularly polarized light beams are emitted from this type of instrument to the measurement surface, the beam splitter that serves the same purpose as PBS2 in Fig. 1, which places the two light beams in close proximity, must be nonpolarizing in nature. This requirement results in an inefficiency of light usage as $75 \%$ of the light will be lost. The high efficiency of light usage in the optical configuration shown in Fig. 1 provides us with an opportunity to use low-power lasers and still achieve optical signals strong enough for electronic phase decoding.

\section{Uniform/Good Wave Front Quality}

Since low operational cost and minimum user adaptations are a design target, the system designed must be able to work on untreated surfaces, which most likely are not of an optical quality surface finish. As lightweight structural applications are the goal, mounting an additional retroreflector to achieve a high-quality optical reflective surface is also out of the question. The two set of optical lenses ( $L 1$, L2, L3, and L4) shown in Fig. 1, all of which are typical glass singlets, are used to eliminate this problem. From a geometric optics viewpoint, ${ }^{14}$ lenses L1 and L3 focus the collimated incident laser light beams emitted from lenses L2 and L4 into point sources on the measurement surfaces, respectively. Therefore, the return light beams after recollimation by the two set of lenses will have the same wave front quality as that of the incident light beams no matter what the measurement surface finish, i.e., the phase information will not be destroyed no matter if the measurement surface is reflective or diffusive. From a Fourier optics viewpoint, ${ }^{15}$ the focusing lens essentially places the measurement surface on the Fourier plane of the incident laser light beam. Therefore, the roughness of the measurement surface will only vary the intensity distribution but not the wave front quality of the light beam after it is reflected/scattered by the measurement surface.

This focused optical configuration also alleviates the stringent constraint on the relative orientations allowed on the two measurement surfaces typically found in standard interferometers. In other words, this new configuration provides us with a way to measure relative displacement of two surfaces whose relative angles are reasonably high. More specifically, a standard interferometer will cease to function when the relative angle of the two measurement surface makes the two optical beams create more than $1 / 4$ waves of tilting fringes whereas the same relative angle will only create a relative shift in position, ${ }^{14,15}$ which will only reduce the signal ratio between the modulation signal and the bias signal. This simple optical arrangement ensures that the designed optical system can operate on an untreated measurement surface, has high flexibility, and can reduced the operating cost in using this system. Furthermore, the unequal focal length adopted on the two optical heads further reduces the background noise and cross talk among the two interference 
arms, as light beams that do not come from the focal spot will not be collected and will not be propagated in the return optical fibers.

\section{Choice of Laser Source}

Since long-term displacement measurement was not the primary goal for this system and the optical path difference of the two interference arms are kept to less than $30 \mathrm{~mm}$, a regular high-quality $\mathrm{He}-\mathrm{Ne}$ laser in lieu of a high-cost frequency/intensity stabilized laser was chosen as the light source. Several issues were considered in choosing the laser source. They included thermal wavelength stability, the coherent length of the laser, and the system phase error generated due to laser wavelength thermal drift. The wavelength stability of a regular $\mathrm{He}-\mathrm{Ne}$ laser is typically better than $2.5 \times 10^{-3}$ $\mathrm{nm} /{ }^{\circ} \mathrm{C}$. In an interferometer configuration, as shown in Fig. 1, with the optical path difference $\Delta L$ between the two interference arms less than $30 \mathrm{~mm}$, the phase error $|\Delta \varphi|$ generated due to optical path difference and a $0.1^{\circ} \mathrm{C}$ laser tube cavity thermal drift within a few minutes is

$$
|\Delta \varphi|=(\Delta L / \lambda) \Delta \lambda / \lambda
$$

With wavelength $\lambda=632.8 \mathrm{~nm}$ for the He-Ne laser, the phase error is around $0.019 \mathrm{rad}$. The corresponding displacement noise generated is then around $0.9 \mathrm{~nm}$, which is within the measurement accuracy. The preceding calculations also indicate that, if a longer displacement measurement stability is needed, several approaches can be adopted. They include using a frequency stabilized laser, further shortening the optical path difference between the two interference arms, and controlling the temperature environment of the laser housing, etc. A regular high-quality $\mathrm{He}-\mathrm{Ne}$ polarized laser typically has a coherent length much longer than $30 \mathrm{~mm}$, therefore, the coherent length requirement does not place a stringent constraint in this newly designed optical system.

\section{Direction Ambiguity}

In order to use optical interferometry for structural dynamic behavior measurements, the so-called directional ambiguity ${ }^{16,17}$ typically present in laser Doppler measurement must be circumvented. A standard two-frequency interferometer approach ${ }^{12}$ was chosen in this design. A Bragg cell was used to shift the laser light frequency by $40 \mathrm{MHz}$ to remove the directional ambiguity of the measurement. $8,9,16,17$ The glass rod shown in Fig. 1 is used to minimize the optical path difference between the two interference arms so as to reduce the phase error induced by the laser thermal wavelength drift effect mentioned earlier. The beam displacer, which was made of two reflective prisms, performs the tasks of maintaining the relative interference arm length and directing the light into the fiber manipulator. The fiber manipulator aligns the incident light beams with respect to the single-mode polarization preserving optical fibers used to guide the light. When aligned properly, these fiber manipulators will have less than a $4 \%$ transmission loss.

\section{Phase and Frequency Decoding}

Since the targeted accuracy for this system is around $1.0 \mathrm{~nm}$ within a typical measurement time span of $10 \mathrm{~min}$ or less, electronic interpretation must be used as $1.0 \mathrm{~nm}$ is much shorter than the visible light wavelength. The interference signals generated by interfering the two returning signals from the two interferometry arms were amplified in the photomultiplier tube. Using the electronic configuration shown in Fig. 1, displacement measurement can be achieved by decoding the relative phase shift between the two beams, and the velocity measurement can be obtained by demodulating the relative frequency shift.

Two different phase demodulation schemes can be used to achieve better than a $1 / 1000$ fringe interpretation. ${ }^{18,19}$ The first phase decoding scheme was obtained by using both the $250-\mathrm{KHz}$ reference signal (i.e., the shifted electronic frequency shown in Fig. 1 is 39.75 $\mathrm{MHz}$ ) and the Doppler shifted $250-\mathrm{KHz}$ signal as the two comparison signals. These two sine waves input were first converted into two square waves to ensure the timing accuracy of the zero crossings. A $24.975-\mathrm{MHz}$ master clock was then used to take a timing measurement on both square wave signals. To obtain one measurement
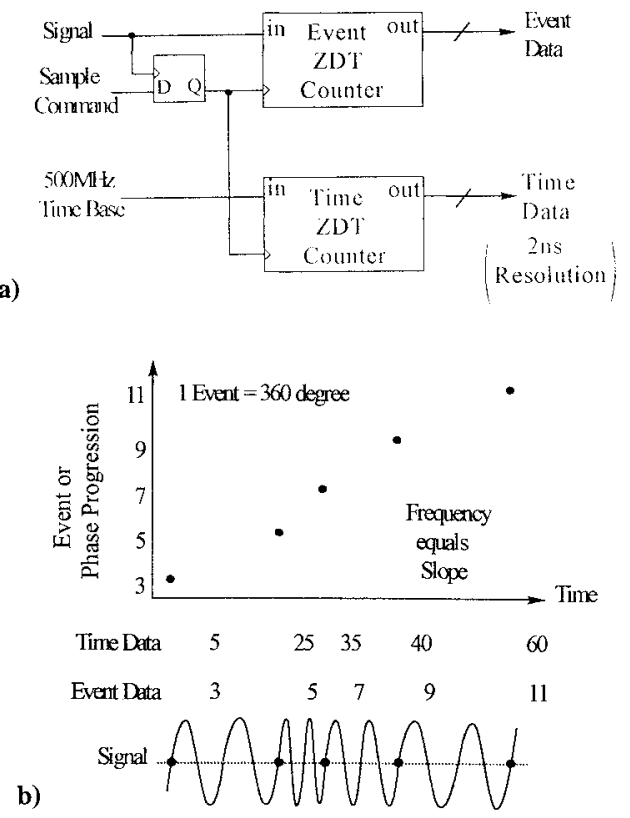

Fig. 2 Configuration of a) simplified phase-digitizing counter and b) phase progression plot.

point 10 measurements were taken and averaged. Since a 24.975 $\mathrm{MHz}$ is 99.9 times that of a $250 \mathrm{KHz}$, the 10 averaged measurements operated as an electronic vernier and allowed the system to produce an output signal at $25 \mathrm{KHz}$ with an accuracy better than a $1 / 1000$ fringe (equals $632.8 \mathrm{~nm} / 2 / 1000=0.3164 \mathrm{~nm}$, if a $\mathrm{He}-\mathrm{Ne}$ laser is used) for a vibration frequency lower than $100 \mathrm{~Hz}$ and with an accuracy better than a $1 / 100$ fringe $(3.164 \mathrm{~nm}$, if a He-Ne laser is used) for a vibration frequency lower than $1 \mathrm{KHz}$.

The second phase demodulation scheme was performed by comparing both the reference signal (with frequency $f$ ) and the Doppler shifted reference signal (with frequency $f+f_{d}$ ). Each signal was sent into a phase-digitizing counter (Fig. 2a), which is basically a combination of two phase digitizers. A phase digitizer is a zerodead-time (ZDT) counter that has an input, into which is fed a signal, such as a sinusoid with some complex phase modulation, represented by $\sin [2 \pi \varphi(t)]$. The phase digitizer output is simply a phase progression, i.e., the output is the instantaneous phase value evaluated at time $t_{i}$ or $\varphi\left(t_{i}\right)$. Although amplitude information is lost in the phase digitizer, this loss of amplitude information presents no degradation effect on the measurement since the measurement signal needed for this newly developed system is completely coded in the phase of the reference signal and the Doppler shifted reference signal. In fact, the removal of the amplitude information significantly reduced signal processing efforts. With each sampling command, a synchronized D-type flip-flop generates an edge which is synchronous with a signal positive transition. This edge was used to trigger both the event and time ZDT counters. Since the signal and trigger are synchronous for the event counter, the event data are exact. The $500-\mathrm{MHz}$ time base shown in Fig. 2a provides us with a timing accuracy of $2 \mathrm{~ns}$. The event count represents the phase progression of the signal, and each event count means the signal has progressed by $2 \pi \mathrm{rad}$. Plotting the phase progression against the time variable along the horizontal axis (Fig. 2b), it is clear that the sampling command and the reference signal frequency $f$ determine how many data points will be taken. Furthermore, since the reference frequency $f$ is much larger than the Doppler frequency $f_{d}$, data points can be obtained between each fringe. The phase accuracy of the fringe interpretation is achieved by the $500-\mathrm{MHz}$ time base. For a $250-\mathrm{KHz}$ Doppler shifted frequency, a $2-\mathrm{ns}$ timing accuracy represents $1 / 2000$ of a fringe. For a He-Ne laser interferometer system, this accuracy represents a resolution of $0.15 \mathrm{~nm}$. The relative displacement between the two measured objects can be obtained by taking the difference between the two phase progression plots.

Two different methods can be used to perform frequency demodulation on the Doppler shifted signal in order to obtain the 


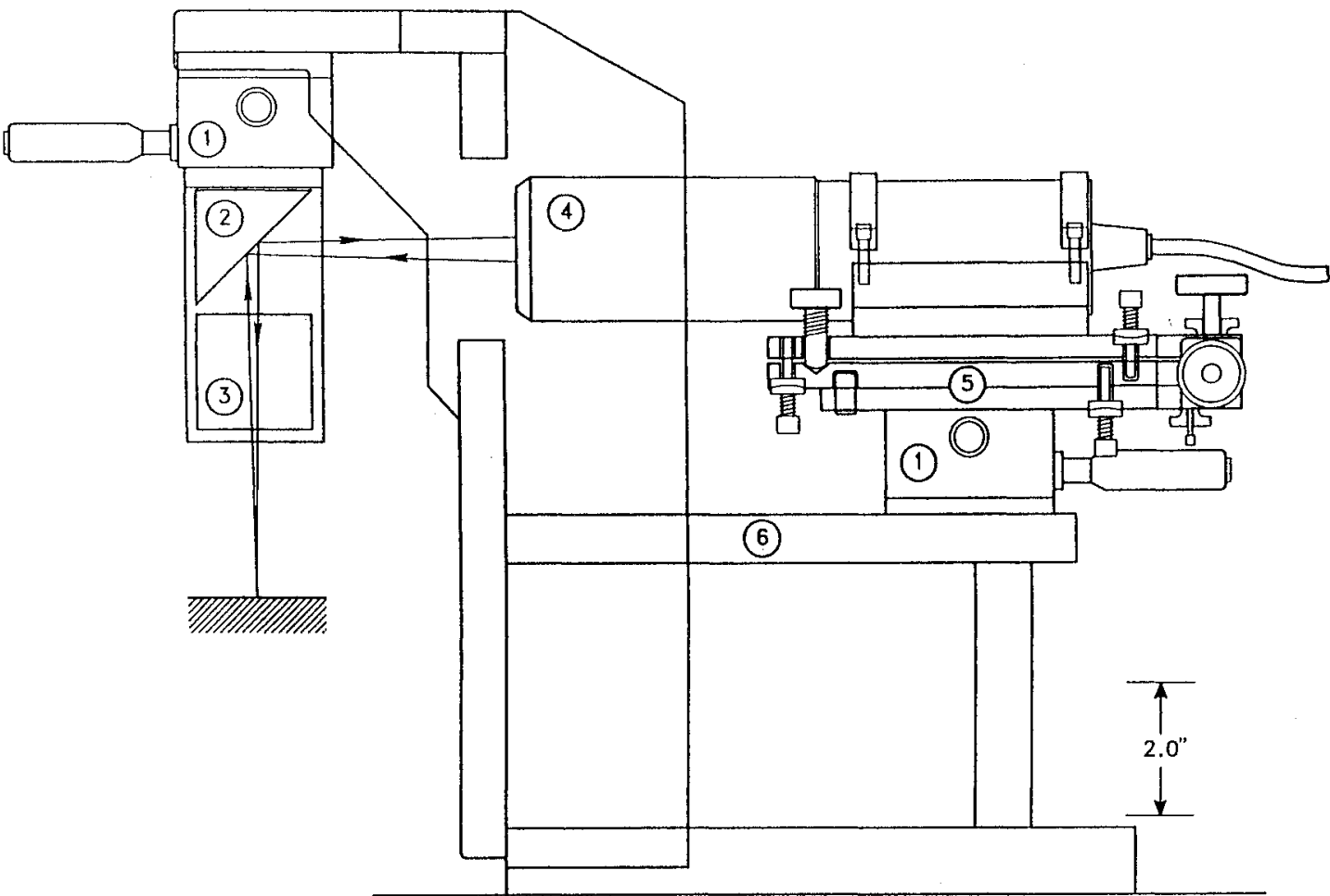

Fig. 3a Side view of the mechanical fixture: 1) XY-translation stage, 2) right angle prism mirror, 3) polarization beam splitter, 4) optical head, 5) tilt-rotation stage, and 6) platform.

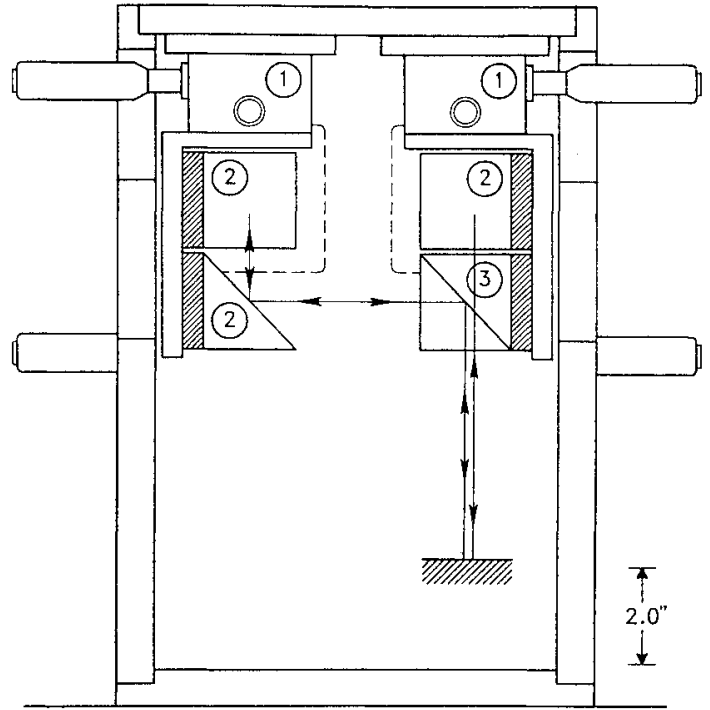

Fig. 3b Front view of the mechanical fixture: 1) XY-translation stage, 2) right angle prism mirror, and 3 ) polarization beam splitter.

velocity signal. The first frequency demodulation scheme is the same as that found in the standard laser Doppler vibrometer (LDV) system, which was achieved by using a tracker. ${ }^{9}$ The second frequency demodulation was obtained by taking the slope of the phaseprogression plot ${ }^{20}$ (Fig. 2b). One thing that should be noted is that the optical signal-to-noise ratio required to perform phase decoding is far more stringent than that of frequency demodulation. Furthermore, the frequency demodulation is much easier to perform than phase decoding. Thus, the experimental results presented later will concentrate primarily on displacement measurement.

\section{Mechanical Fixtures}

Relative thermal deformation between the mounting fixtures for the two interference arms make a significant portion of the measurement noise. Taking aluminum 6061-T6 as an example, the thermal expansion coefficient is $23 \times 10^{-6} /{ }^{\circ} \mathrm{C}$. If the mechanical length of the two supporting structures is different by $10 \mathrm{~mm}$, a $1^{\circ} \mathrm{C}$ relative thermal deformation will create a measurement error in the range of $230 \mathrm{~nm}\left(=1 \times 23 \times 10^{-6} \times 10 \times 10^{-3} / 10^{-9} \mathrm{~nm}\right)$. Therefore, either the testing environment must be controlled precisely or the thermal mass of the supporting fixtures must be large enough to prevent rapid thermal fluctuations. Alternatively, the supporting fixture must have a balanced configuration to minimize the relative mechanical thermal deformation noise. A novel mechanical fixture, as shown in Fig. 3 that supports the optical system and is characterized by easy alignment and minimal relative thermal deformation between the two interferometry arms, was also designed as part of this testing system. Both optical heads are located on the same stainless-steel platform to ensure that temperature variation does not cause significant relative thermal deformation. The multiple axes alignment capabilities of the optical head supporting stage provides us with a way of measuring the two object surfaces that are not exactly parallel to the mounting platform. The polarization beam splitter, right-angle prism mirrors, are supported in such a way that the separation distance between the two measurement spots can be varied from zero to more than $5.0 \mathrm{in}$. To increase its structural rigidity and to raise the structural resonant frequency in order to perform highfrequency vibration measurements, the mechanical fixture was also designed using a minimum number of stages in order to obtain a low-profile design.

\section{Experimental Results}

This newly developed differential interferometer/vibrometer was tested using the first phase decoding scheme. An experimental slider was used to examine the accuracy of this newly developed system. This slider was first fly tested on a glass disk using white light interferometry. ${ }^{20-23}$ Then the slider was flown on a thin-film disk with an approximate 40 -nm disk surface roughness. First, the spacing curves (relative slider/disk spacing vs the disk linear velocity) of three separate points that were not colinear on the slider $(Z 1, Z 2$, and $Z 3$ ) were obtained. Then the spacing curve $Z 4$, which corresponds to the flying height of the magnetic head location measured by white light interferometry, i.e., the relative spacing at the middle of the trailing edge of this experimental slider, was calculated by assuming that the flying slider was a rigid body in our measurement frequency range (Fig. 4). More specifically, once the spacing curves 


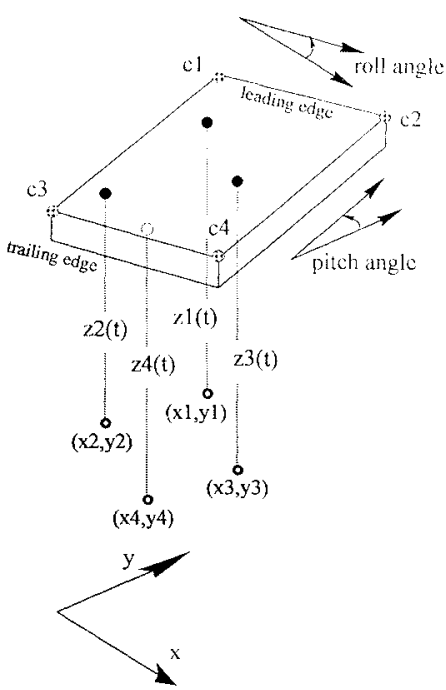

Fig. 4 Spacing curve computation on an arbitrary point of a flying slider using three measured spacing curves.

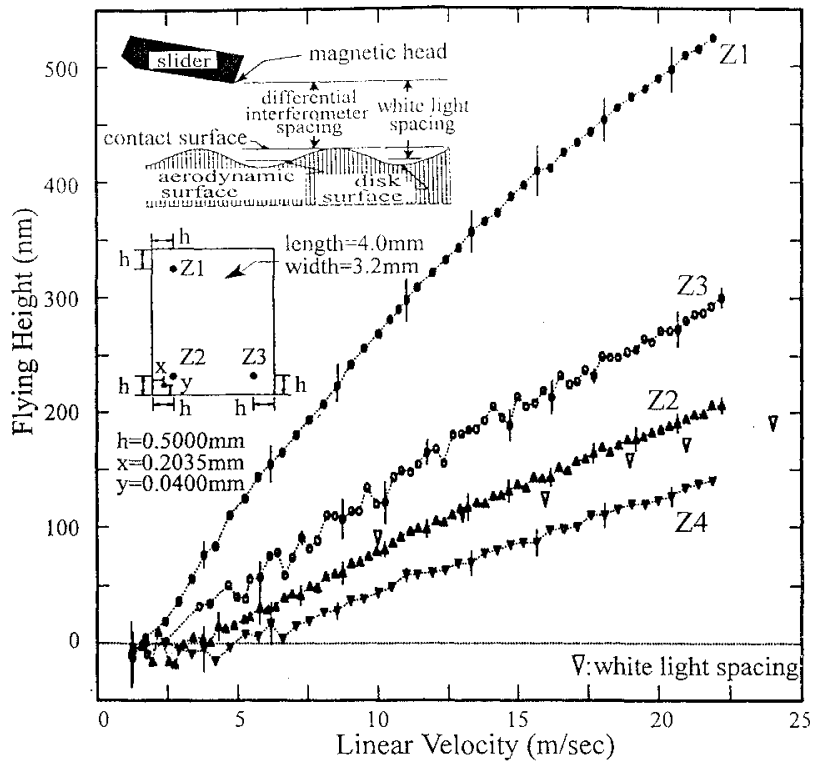

Fig. 5 Comparison of a flying height measurement obtained by white light interferometry to one obtained by differential interferometer/vibrometer.

of the three separate points are measured, the fourth point on the slider can be calculated as follows:

$$
Z 4=Z 1+\left\{\left[\frac{-1}{N_{3}}\right] \times\left[\left(N_{1} \times \Delta x_{41}\right)+\left(N_{2} \times \Delta y_{41}\right)\right]\right\}
$$

where

$$
\begin{aligned}
& N_{1}=\left[\left(\Delta y_{21} \times \Delta Z_{31}\right)\right]-\left[\left(\Delta y_{31} \times \Delta Z_{21}\right)\right] \\
& N_{2}=\left[\left(\Delta Z_{21} \times \Delta x_{31}\right)\right]-\left[\left(\Delta Z_{31} \times \Delta x_{21}\right)\right] \\
& N_{3}=\left[\left(\Delta x_{21} \times \Delta y_{31}\right)\right]-\left[\left(\Delta x_{31} \times \Delta y_{21}\right)\right]
\end{aligned}
$$

and $\Delta x_{i j}=x_{j}-x_{i}, \Delta y_{i j}=y_{j}-y_{i}$, and $\Delta Z_{i j}=Z_{j}-Z_{i}$, with $i, j=1,2,3,4$. As the dimensions of current sliders are less than $4 \times 3 \mathrm{~mm}$ and our goal is to limit computation errors to less than a nanometer, the coordinate accuracy of the three data points whose spacing curves are to be measured must be in the range of $20 \mu \mathrm{m}$. Therefore, a long-range microscope was implemented in order to obtain the required coordinate measurement accuracy.

As is shown in Fig. 5, the flying height of the slider measured by white light interferometry and the spacing of the slider measured by differential interferometer should have a datum shift $\Delta d_{r}$ close to the disk roughness. It is also clear from Fig. 5 that the datum shift between the data obtained by the white light interferometry and by a)
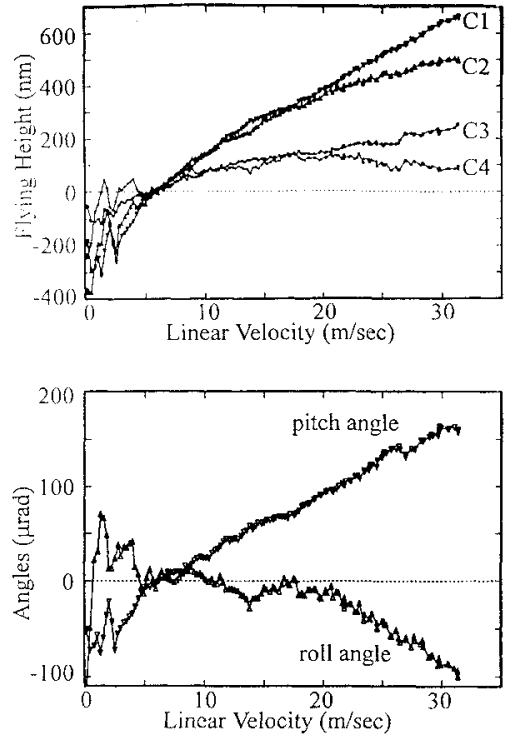

Fig. 6 Experimental slider: a) spacing curves at four corners and b) pitch/roll angles.

the newly developed differential interferometer is also around 40 $\mathrm{nm}$, the measured disk roughness. Additionally, we can see from Fig. 5 that no data points are obtained by white light interferometry when the fly height is less than $75 \mathrm{~nm}$. This is the so-called $3-\mu$ in. $(75-\mathrm{nm})$ barrier currently limiting the use of white light interferometry technique ${ }^{23}$ used in the disk drive industry. It is also clear that the newly developed differential interferometry system has capabilities to measure displacement down to a few nanometers. This data verifies the design principles and touches upon some of the capabilities of the newly developed system. In comparison to some commonly used small displacement measurement techniques, such as capacitive or inductive type of detectors, this newly developed interferometer avoids the need to prepare special testing samples. As industrial development processes get more and more driven by the pressure of time to market and low cost, this added advantage will become even more valuable.

By using this newly developed differential interferometer, both the dynamic and steady-state behavior of the flying sliders relative to the disk can be measured. For example, by measuring the spacing curves of three separate points on top of an experimental slider (Fig. 4) relative to a specific point on top of the disk for all disk spinning speeds, we can obtain the steady-state altitude of the flying slider with respect to the disk at different linear disk velocities. This is achieved by sampling the spacing curves at a predetermined spindle encoder pulse. Since the encoder pulse is fixed relative to a point on top of the disk after it is mounted, the flying height data sampling will be fixed with respect to a fixed point on top of a disk when the sampling is triggered by a fixed encoder pulse. More specifically, the sampling frequency continues to vary when a disk starts at zero speed, then gradually increases speed until it arrives at full speed (called spin up) and then finally gradually reduces speed back to zero (called spin down). From Fig. 6, we can see the relative spacing curves of the four corners (c1, c2, c3, and c4) and the relative pitch/roll angles of this slider. Since this newly developed interferometer measures only the relative displacement of two surfaces but not the absolute distance between any two surfaces, the so-called zero flying height point must be determined by other means. An acoustical emission signal, whose strength is correlated to the interaction between the slider and the disk, was used to identify the point that all four corners of the sliders left the disk surface, i.e., the socalled zero spacing point. The data analysis algorithm adjusts the relative flying height signals obtained for all four corners so that the zero spacing points are coincident with each other. The positive or negative flying heights are, thus, all measured relative to the zero spacing points. Similarly, the relative pitch/roll angles obtained are set to zero at the zero spacing points. This type of information not only is invaluable to the understanding of slider/disk interaction 


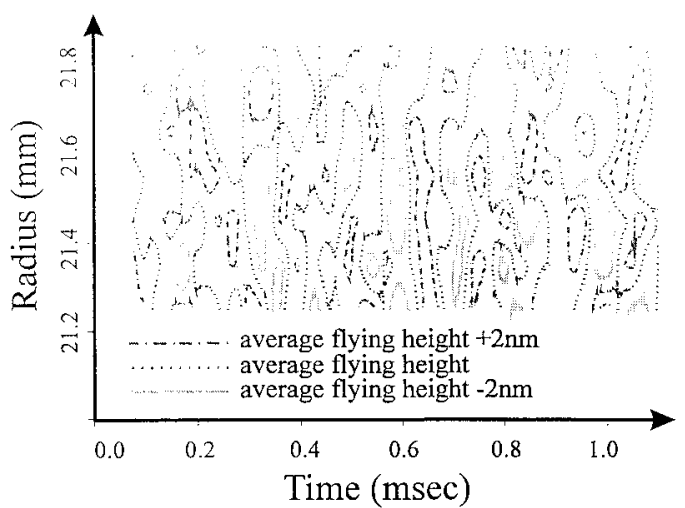

Fig. 7 Spacing modulation curves at a region of a spinning disk.

behaviors but also is important for shortening the development cycle since no specialty slider/disk pairs needs to be prepared.

The slider/disk spacing modulation data relative to a region of the spinning disks can also be measured by using this interferometer (Fig. 7). After triggering at a specific spindle encoder plus, 2000 data points were taken at a constant sampling rate along a specific radius $r$, say, $r=21.4 \mathrm{~mm}$. This process was repeated every 0.1 mm along the radius of the disk. Finally, all data points with a higher than 12,000 total were used to create the flying height modulation contour plot, which indicates how slider flying height is modulated with respect to the mean flying height. The high bandwidth nature of this measurement demonstrates the dynamic capabilities of this newly developed interferometer.

\section{Conclusions}

High-performance measurement systems are an integral part of any new technology/product development. As mission requirements of mechanical structures, either in aerospace structures or other highperformance/high-precision mechanical systems, become ever more demanding, sensor (measurement systems) performance faces an ever more increasing emphasis on continued improvement. Since nanometer-types of measurements are becoming common testing tasks, measurement systems similar to the newly developed differential laser interferometer/vibrometer system will be needed.

By adopting a series of innovative design approaches, this newly developed differential laser interferometer/vibrometer achieves design goals such as nanometer displacement measurement accuracy, no requirement to have an optical quality measurement surface, ability to handle differential as well as absolute displacement/velocity measurement, and high efficiency in laser light usage. It is clear from the design considerations discussed in this paper that optical and mechanical integration are indispensable in this type of highprecision measurement system development. The performance and measurement capabilities of this newly developed system have been verified by comparing the testing results to those obtained by white light interferometry techniques on a slider/disk system.

\section{References}

${ }^{1}$ Anderson, E., "Testing and Application of a Viscous Passive Damper for Use in Precision Truss Structures," Proceedings of the AlAA/ASME/ASCE/AHS/ASC 32nd Structures, Structural Dynamics, and
Materials Conference (Baltimore, MD), AIAA, Washington, DC, 1991, pp. 2796-2808 (AIAA Paper 91-0966).

${ }^{2}$ Miller, D., Blackwood, G., Jacques, R., Hyde, T., and Kim, E., "The MIT Multipoint Alignment Testbed: Technology Development for Optical Interferometry," Society of Photo-Optical Instrumentation Engineers Conf. on Active and Adaptive Optical Components, San Diego, CA, July 1991.

${ }^{3}$ Laskin R., and San Martin, M., "A Spaceborn Imaging InterferometerThe JPL CSI Mission Focus," Third NASA/Dept. of Defense Control Structure Interactions (CSI) Technology Conf., San Diego, CA, Jan.-Feb. 1989.

${ }^{4}$ Lee, C.-K., and Wu, T.-W., "Differential Laser Interferometer/Vibrometer," IBM Technical Disclosure Bulletin, Vol. 35, No. 1A, 1992, pp. $189-195$.

${ }^{5}$ Davidson, R., "Interferometer Apparatus and Method for Determining the Spatial Relationship of Two or More Objects," US Patent No. 2,681,447, July $21,1987$.

${ }^{6}$ Zhu, L.-Y., and Bogy, D. B., "Head-Disk Spacing Fluctuation Due to Disk Topography in Magnetic Recording Hard Disk Files," Society of Tribologists and Lubrication Engineers (STLE) Tribology and Mechanics of Magnetic Storage Systems, No. SP-26, 1989, pp. 160-167.

7 Anon., "5501A Laser Transducer System," Hewlett-Packard Corp., Santa Clara, CA, May 1983.

${ }^{8}$ Drain, L. E., The Laser Doppler Technique, Wiley, New York, 1980.

${ }^{9}$ Anon., "55N20 Doppler Frequency Tracker Institution Manual," Dantec Electronics, Inc., Mahwah, NJ, 1981.

${ }^{10}$ Anon., "Fiber Optical Laser Vibrometer," Polytec Optronics, Inc., Costa Mesa, CA, 1991.

${ }^{11}$ Barker, A. J., Jaeger, P. E., and Oliver, D. E., "Non-contacting Vibration Measurement: Role in Design and Industrial Applications," Stress and Vibration: Recent Developments in Industrial Measurement and Analysis, Vol. 1084, Society for Photo-Optical Instrumentation Engineers, Bellingham, WA, 1989, pp. 293-299.

${ }^{12}$ Augustyn, W., and Davis, P., "An Analysis of Polarization Mixing Errors in Distance Measuring Interferometers," Journal of Vacuum Science Technology B, Vol. 8, No. 6, 1990, pp. 2032-2036.

${ }^{13}$ Riener, T., Goding, A. C., and Talke, F. E., "Measurement of Head/Disk Spacing Modulation Using a Two Channel Fiber Optic Laser Doppler Vibrometer," IEEE Transactions on Magnetics, Vol. 24, No. 6, 1988, pp. 2745-2747.

${ }^{14}$ Hecht, E., Optics, 2nd ed., Addison-Wesley, Menlo Park, CA, 1990.

${ }^{15}$ Goodman, J. W., Introduction to Fourier Optics, Mc-Graw-Hill, New York, 1968

${ }^{16}$ Buchhave, P., "Laser Doppler Velocimeter with Variable Optical Frequency Shift," Optics and Laser Technology, Feb. 1975, pp. 11-16.

${ }^{17}$ Durst, F., and Zare, M., "Removal of Pedestals and Ambiguity of Optical Anemometer Signals," Applied Optics, Vol. 13, No. 11, 1974, pp. 2562-2579.

${ }^{18}$ Anon., "OPTRAMETER Measurement Module Operator's Manual," Dantec Electronics Inc., Allendale, NJ, 1989.

${ }^{19}$ Anon., "Bringing a New Dimension to Measurement Analysis," HP 5371A Frequency and Time Interval Analyzer Data Sheet/Brochure, Hewlett-Packard Instrument Div., Literature No. 5952-7940D, North Hollywood, CA, 1988.

${ }^{20} \mathrm{Lin}, \mathrm{C}$., "Technique for the Measurement of Air-Bearing Separation-A Review," IEEE Transactions on Magnetics, Vol. MAG-9, No. 4, 1973, pp. 673-677.

${ }^{21}$ Lin, C., and Sullivan, R. F. "An Application of White Light Interferometry in Thin Film Measurement," IBM Journal of Research and Development, Vol. 16, No 3, 1972, pp. 269-276.

${ }^{22}$ Best, G. L., Horne, D. E., Chiou, A., and Sussner, H., "Precise Optical Measurement of Slider Dynamics," IEEE Transactions on Magnetics, Vol. MAG-22, No. 5, 1986

${ }^{23}$ Anon., "Automatic Digital Flying Height Tester," Pacific Precision Lab., Inc., Chatsworth, CA, 1988. 